\title{
Gender differences in atrial fibrillation risk factors, presentation, and management in Tabuk, Kingdom of Saudi Arabia
}

\author{
Abdullah Sahban Alshamrani ${ }^{1}$, Bader Dhafer Alqarni ${ }^{1}$, Hyder Osman Mirghani ${ }^{2}$
}

\author{
${ }^{1}$ Faculty of Medicine, University of Tabuk, Tabuk, Saudi Arabia \\ ${ }^{2}$ Associate Professor of Internal Medicine and Endocrine, Medical Department, Faculty of Medicine, University of \\ Tabuk, Tabuk, Saudi Arabia
}

Type of article: Short report

\begin{abstract}
Background: There is a lack of data regarding gender differences in atrial fibrillation presentation and clinical characteristics in Saudi Arabia.

Objective: To assess gender differences in atrial fibrillation risk factors, presentation, and management strategy in Tabuk.

Methods: This retrospective cross-sectional study was conducted in King Fahad Specialist Hospital in Tabuk City, Saudi Arabia. The medical records of all patients with atrial fibrillation in the years 2016 and 2017 were approached using a structured questionnaire to collect demographic factors, atrial fibrillation risks including diabetes, hypertension, ischemic heart disease, valvular heart disease, respiratory diseases (Bronchial Asthma, chronic obstructive airways disease, and pulmonary fibrosis), cardiomyopathy, thyrotoxicosis, and congenital heart disease. The different types of atrial fibrillation, management strategy, and complications were also reported. IBM-SPSS version 20 was used for data analysis. The Chi-square test was applied to compare men and women.

Results: There were 93 patients with atrial fibrillation (59.2\% males), hypertension, diabetes, ischemic heart disease, cardiomyopathy, thyrotoxicosis, congenital heart disease, and valvular heart disease was evident in $72 \%$, $31.2 \%, 32.1 \%, 9.7 \%, 2.2 \%, 2.2 \%$, and $10.7 \%$ respectively, while respiratory diseases, chronic atrial fibrillation, rate control strategy, and stroke were reported in $7.6 \%, 70.9 \%, 68.8 \%$, and $16.1 \%$ of patients respectively. Women were older and had more paroxysmal atrial fibrillation with significant statistical differences $(\mathrm{p}=0.010)$. No significant differences were evident regarding other characteristics.

Conclusion: No significant differences were found between males and females except age and paroxysmal atrial fibrillation, which were higher among women.

Keywords: Gender difference, Atrial fibrillation, Saudi Arabia
\end{abstract}

\section{Introduction}

Atrial fibrillation (AF) is the most common sustained abnormal heart rhythm, or arrhythmia, worldwide. The likelihood of developing AF increases with age. The prevalence is $0.4 \%$ in the general population, $3-5 \%$ in those $>65$ years and $10 \%$ in in subjects aged >eighty years (1). This dangerous arrhythmia is often associated with serious diseases including coronary heart disease, diabetes, hypertension, and heart failure (2). Serious complications like stroke and peripheral arterial disease may happen from thromboembolism. In the Kingdom of Saudi Arabia, hypertension is the most common risk factor followed by ischemic heart disease and smoking (3). AF is prevalent worldwide; it affects more than six million people in Europe. These numbers are projected to increase 2.5 -fold by 2050 due to an aging population, improved survival of people with conditions which predispose AF (e.g., heart

\section{Corresponding author:}

Associate Professor Dr. Hyder Osman Mirghani, Medical Department, Faculty of Medicine, University of Tabuk, Tabuk, Saudi Arabia. Tel: +966.569344569Email: s.hyder63@hotmail.com

Received: February 14, 2018, Accepted: April 28, 2018, Published: March 2019

iThenticate screening: April 28, 2018, English editing: June 09, 2018, Quality control: July 12, 2018

This article has been reviewed / commented by three experts

Ethics approval: University of Tabuk (Ref: 30.304, Date: July 09, 2017)

(C) 2019 The Authors. This is an open access article under the terms of the Creative Commons Attribution-NonCommercialNoDerivs License, which permits use and distribution in any medium, provided the original work is properly cited, the use is non-commercial and no modifications or adaptations are made. 
attack), and a higher incidence of AF itself. Alarmingly, AF is underdiagnosed and under-treated even though it causes severe strokes, many of which can be prevented (4). Literature is scarce on this morbid arrhythmia in the Kingdom of Saudi Arabia (5). The prevalence of atrial fibrillation risk factors including hypertension, diabetes mellitus, and ischemic heart disease are on the rise in the Kingdom of Saudi Arabia, while rheumatic heart disease is not uncommon (6). Gender differences in atrial fibrillation risk factors and outcomes have been discussed in previous literature with conflicting results, with some reporting a higher risk of stroke (7) and others reporting lower bleeding tendencies (8) among women. Less evidence is available on the complex interaction between atrial fibrillation, gender, heart failure, and ischemic heart disease (9). To our best of knowledge, no researchers have studied the gender differences in atrial fibrillation in Saudi Arabia in spite of a large proportion of the population at risk of this serious health problem. There is a need for recent data to identify the gender differences of the risk factors of this common cardiac arrhythmia for prevention and proper management when appropriate. Thus, we conducted this research to study the gender differences in atrial fibrillation risk factors, presentation and management in Tabuk, the Kingdom of Saudi Arabia.

\section{Material and Methods}

\subsection{Research design and patient selection}

This cross-sectional retrospective study was conducted at King Fahad Specialist Hospital. The patients' records from the cardiology unit during the years 2016 and 2017 were approached confidentially and the personal data and privacy was secured. All the patients above 18 years with atrial fibrillation were included and others with incomplete data were excluded.

\subsection{Data collection}

Atrial fibrillation was diagnosed by cardiologists depending on clinical findings or irregular pulse and a pulse deficit of $>15$, the diagnosis was confirmed by electrocardiogram according to the American College of Cardiology Guidelines (10). The data were collected by the first and second authors. A structured questionnaire was used, and the following information was collected: Age, sex, the risks of atrial fibrillation including ischemic heart disease, diabetes, hypertension, thyrotoxicosis, cardiomyopathy, valvular and congenital heart disease, and cigarette smoking. The types of atrial fibrillation (acute, chronic, persistent, or paroxysmal), type of therapy used including rate or rhythm control, cardiac pacing, and anticoagulation were also collected. The associate's respiratory diseases like bronchial asthma, pulmonary fibrosis, and chronic obstructive airway disease were also reported, as were stroke and peripheral arterial disease.

\subsection{Ethics of research}

The ethical committees of the Medical College, University of Tabuk, and King Fahad Specialist Hospital approved the research (Ref: 30.304, Date: July 09, 2017).

\subsection{Statistical analysis}

The Statistical Package for Social Sciences IBM(C) SPSS $\odot$ version 20 (IBM(C Corp, Armonk, NY, USA) was used for data analysis; the data were presented as percentages or mean \pm SD unless otherwise specified. The Chi-square test was used to compare women and men. A p-value of $<0.05$ was considered significant.

\section{Results}

There were 93 patients with atrial fibrillation, their ages mean \pm SD $(64.60 \pm 16.10)$ years, $59.8 \%$ were women. Table 1 depicts the basic clinical characteristics in which the majority (72\%) were hypertensive, nearly one third were affected by diabetes, cardiomyopathy was evident in $9.7 \%, 12.9 \%$ were cigarettes smokers, $2.2 \%$ had thyrotoxicosis and congenital heart disease, while recreational drug use was reported in $1.1 \%$. In the present study, the majority $(70.9 \%)$ had chronic atrial fibrillation, $11.8 \%$ had acute atrial fibrillation, and atrial fibrillation was persistent in only a minority $(1.1 \%$ ), while paroxysmal atrial fibrillation was reported in $10.7 \%$ of patients (Table 2 ). The present data showed ischemic heart disease in $31.2 \%$, valvular heart disease in $10.7 \%$ of patients, while cardiomyopathy was demonstrated in $9.7 \%$ (Table 2). Regarding respiratory disease, bronchial asthma was found in $5.6 \%$, lung fibrosis in $1.1 \%$, and chronic obstructive airway was not reported among the study group (Table 2). In the current study, the majority $(68.8 \%)$ of patients with atrial fibrillation received rate control therapy, rhythm control was shown in $11.8 \%, 9.7 \%$ were on anticoagulation drugs, and one patient had a cardiac pacemaker (Table 2). Table 2 shows vascular complication among patients with atrial fibrillation, in which 16.1 developed strokes and $2.2 \%$ developed a peripheral vascular injury. In the present study, men were younger than women with significant statistical difference (59.56 \pm 19.12 vs. $59.56 \pm 19.12, \mathrm{p}=0.016)$. No significant statistical differences were evident regarding diabetes 
http://www.ephysician.ir

(32.4\% vs. $29.6 \%, p=0.776)$, hypertension $(62.1 \%$ vs. $79.6 \%, p=0.067)$, thyrotoxicosis $(2.7 \%$ vs. $1.9 \%, p=0.786)$, valvular heart disease $(8.1 \%$ vs. $11.1 \%, \mathrm{p}=0.637)$, ischemic heart disease $(37.8 \%$ vs. $25.9 \%, \mathrm{p}=0.277)$, and paroxysmal atrial fibrillation $(16.2 \%$ vs. $7.4 \%, p=0.306)$, while chronic atrial fibrillation was commoner among females with significant statistical difference $(81.5 \%$ vs. $56.7 \%, \mathrm{p}=0.010)$ (Table 3 ).

Table 1. Age, sex, family history of atrial fibrillation, and atrial fibrillation risk factors among patients with atrial fibrillation

\begin{tabular}{|l|l|l|}
\hline Character & $\mathrm{n}(\%) /($ mean \pm SD) \\
\hline Sex & Males & $55(59.2)$ \\
\hline Diabetes & $38(40.2)$ \\
\hline Hypertension & $29(31.2)$ \\
\hline Thyrotoxicosis & $67(72)$ \\
\hline Cardiomyopathy & $2(2.2)$ \\
\hline Congenital heart disease & $9(9.7)$ \\
\hline Smoking & $2(2.2)$ \\
\hline Ischemic heart disease & $12(12.9)$ \\
\hline Valvular heart disease & $29(31.2)$ \\
\hline Recreational drugs & $10(10.7)$ \\
\hline Family history of atrial fibrillation & $1(1.1)$ \\
\hline Age (mean \pm SD) & $0(0.0)$ \\
\hline
\end{tabular}

Table 2. Clinical and paraclinical features of the patients

\begin{tabular}{|l|l|l|}
\hline Variables & $\mathrm{n}(\%)$ \\
\hline Types of atrial fibrillation & Acute & $11(11.8)$ \\
\cline { 2 - 3 } & Chronic & $66(70.9)$ \\
\cline { 2 - 3 } & Persistent & $1(1.1)$ \\
\cline { 2 - 3 } & Paroxysmal & $10(10.7)$ \\
\hline Echocardiography finding & Valvular heart disease & $10(10.7)$ \\
\cline { 2 - 3 } & Ischemic heart disease & $29(31.2)$ \\
\cline { 2 - 3 } & Cardiomyopathy & $9(9.7)$ \\
\hline Respiratory diseases among patients with atrial fibrillation & COPD & $0(0.0)$ \\
\cline { 2 - 3 } & Bronchial asthma & $6(6.5)$ \\
\cline { 2 - 3 } & Lung fibrosis & $1(1.1)$ \\
\hline Modalities of therapy among patients with atrial fibrillation & Rate control & $64(68.8)$ \\
\cline { 2 - 3 } & Amiodarone & $11(11.8)$ \\
\cline { 2 - 3 } & Pacemaker & $1(1.1)$ \\
\cline { 2 - 3 } & Anticoagulation & $9(9.7)$ \\
\hline Vascular complications & Stroke & $15(16.1)$ \\
\cline { 2 - 3 } & Peripheral arterial disease & $2(2.2)$ \\
\hline
\end{tabular}

Table 3. A comparison between males $(n=37)$ and females $(n=54)$ patients with atrial fibrillation

\begin{tabular}{|l|l|l|l|}
\hline Character & Males; $\mathrm{n}(\%) /($ mean \pm SD) & Females; $\mathrm{n}(\%) /(\mathrm{mean} \pm \mathrm{SD})$ & $\mathrm{p}$-value* \\
\hline Age $($ mean \pm SD & $59.56 \pm 19.12$ & $67.74 \pm 12.78$ & 0.016 \\
\hline diabetes & $12(32.4)$ & $16(29.6)$ & 0.776 \\
\hline Hypertension & $23(62.1)$ & $43(79.6)$ & 0.067 \\
\hline Thyrotoxicosis & $1(2.7)$ & $1(1.9)$ & 0.786 \\
\hline Chronic atrial fibrillation & $21(56.7)$ & $44(81.5)$ & 0.010 \\
\hline Valvular heart disease & $3(8.1)$ & $6(11.1)$ & 0.637 \\
\hline Ischemic heart disease & $14(37.8)$ & $14(25.9)$ & 0.277 \\
\hline Paroxysmal atrial fibrillation & $6(16.2)$ & $4(7.4)$ & 0.306 \\
\hline Rate control strategy & $24(64.9)$ & $13(34.1)$ & 0.445 \\
\hline Anticoagulation use & $3(8.1)$ & $39(72.2)$ & 0.673 \\
\hline Crebrovascular disease & $3(8.1)$ & $11(20.4)$ & 0.111 \\
\hline
\end{tabular}

*Chi square test 


\section{Discussion}

Atrial fibrillation is a common serious health problem, it is associated with various diseases and risk factors. In the current study, the majority of patients were hypertensive and nearly one third were affected by diabetes and ischemic heart disease. The current findings were similar to those of Hersi et al. (3) who concluded hypertension in $63.3 \%$, and diabetes mellitus in $48 \%$. A recent review and meta-analysis (11) drew the conclusion that rhythm control strategy seems to significantly increase the adverse events compared to rate control, which is the preferred treatment of most patients with atrial fibrillation unless there are unbearable symptoms or the patient is hemodynamically unstable or there are other specific reasons for the introduction of rhythm control. In the present data, rate and rhythm control were observed in $68.8 \%$ and $12.9 \%$ respectively and in line with current evidence. Similarly, Hersi and colleagues (3) observed that the rate control was the commonest management strategy (66.2\%), while rhythm control was found in $12 \%$ and were in accordance to the present observation. The higher rate of smoking among the Saudi atrial fibrillation survey $(23.5 \%$ vs. $12.9 \%$ in the current data) could be explained by the difference in the sample size or the conservative nature of the present sample. Atrial fibrillation is a heterogeneous disorder; the clinical pattern varies according to the type of care provided. A study conducted in Spain (12) observed permanent atrial fibrillation in $51 \%$ of those admitted to the internal medicine department and $30 \%$ of the patients admitted to the cardiology department. In the present study, acute and chronic atrial fibrillation were present in $11.8 \%$ and $70.9 \%$ respectively, similar results were observed in a study conducted in the central region of Saudi Arabia (4) which found acute atrial fibrillation in $21.8 \%$ and chronic atrial fibrillation in $78.1 \%$. The lower rate of chronic pulmonary diseases (bronchial asthma, chronic obstructive pulmonary disease, and idiopathic pulmonary fibrosis) in the current study (7.7\% in the current data compared to $31.8 \%$ in the previous study) could be due to a higher rate of risk factors including cigarette smoking, the atmospheric pollution in central Saudi Arabia or familial factors. In the current study, 9.7\% of patients were on anticoagulants, which was lower than in other studies (12). The different treating physicians and the indication for anticoagulation could explain the differences, but in the face of relatively higher cerebrovascular (16.1\%) and valvular heart disease $(10.7 \%)$ in the present sample, there could be an under prescription of anticoagulants. The lower rate of valvular heart disease in our survey $(10.7 \%$ vs. $23.6 \%$ in the central region of Saudi Arabia) could be explained by the higher rate of rheumatic disease in that region and the earlier time of the study which was conducted in 2011. A large-scale study conducted in 26 countries (13) concluded paroxysmal atrial fibrillation in $26.5 \%$, persistent atrial fibrillation in $23.8 \%$, while permanent atrial fibrillation was found in $49.6 \%$, which were in contradiction to our study. Plausible explanations could be the difference in the sample size and the fact that the previous study was a real-life survey. However, the rates of cerebrovascular disease observed were slightly lower than the present study $(11.7 \%$ vs. $16.1 \%)$. In the present study, men were generally younger than women, in similarity to Schnabel et al. (14). In the current survey, chronic atrial fibrillation was commoner among men, and while there were no significant statistical differences between males and females regarding paroxysmal atrial fibrillation and hypertension, the present findings are in contradiction to Potpara et al. (15), who observed a higher rate of paroxysmal atrial fibrillation and hypertension among females. The younger age of the previous study sample, as well as race and sample size, could explain the differences. The EUR Observational Research Programme pilot survey on atrial fibrillation (16) found no differences across gender regarding hyperthyroidism, diabetes mellitus, type of atrial fibrillation, oral anticoagulants use, rate control strategy, or previous stroke, similar to the present findings, however, the study observed a higher rate of valvular heart disease and lower rate of coronary artery disease among females, in contradiction to the present findings in which no significant differences were evident between men and women. The present findings are consistent with a recent study (16) which concluded no gender difference regarding stroke, and use of anticoagulation. The study observation of a higher use of rate control strategy is in similarity to the current finding. In the current study, females were eight years elder than males and had more chronic atrial fibrillation compared to men which is in line with the study of Schnabel et al. (13) who found similar results. Schnabel et al. concluded similar prescription rates of anticoagulation drugs among males and females in accordance with the current findings. The current data showed a similar rate of stroke between males and females similar to the previous study (13). During the previous years, an increasing rate of atrial fibrillation, introduction of new anticoagulation in particular non-vitamin K antagonists, and new guidelines for atrial fibrillation have been witnessed, which may explain the discrepancy between the current findings and previous research. The limitations of the study were the retrospective nature of the study, the small size of the sample, and the fact that the study was conducted at a single tertiary center, so generalization cannot be assured.

\section{Conclusions}

Females were older than males. No differences were found between males and females regarding atrial fibrillation presentation and management apart from paroxysmal fibrillation, which was commoner among women. The current 
findings pointed to the high rate of paroxysmal atrial fibrillation among women and the need for home monitoring in case this morbid arrhythmia is suspected because normal electrocardiogram cannot exclude atrial fibrillation. Further prospective studies assessing home ECG monitoring for paroxysmal atrial fibrillation diagnosis are needed to prevent deleterious consequences of this common arrhythmia including stroke and cognitive impairment.

\section{Acknowledgments:}

This is study was self-funded and conducted at King Fahad Specialist Hospital. We would like to acknowledge Dr. Salem AlBalawi, Consultant Cardiologist and the head of the Department of Cardiology, King Fahad Specialist Hospital.

\section{Conflict of Interest:}

There is no conflict of interest to be declared.

\section{Authors' contributions:}

All authors contributed to this project and article equally. All authors read and approved the final manuscript.

\section{References:}

1) Piccinocchi G, Laringe M, Guillaro B, Arpino G, Piccinocchi R, Nigro G, et al. Diagnosis and management of atrial fibrillation by primary care physicians in Italy: a retrospective, observational analysis. Clin Drug Investig. 2012; 32(11): 771-7. doi: 10.1007/s40261-012-0005-5. PMID: 23018284 PMCID: PMC3693438.

2) Lip GY, Laroche C, Dan GA, Santini M, Kalarus Z, Rasmussen LH, et al. A prospective survey in European Society of Cardiology member countries of atrial fibrillation management: baseline results of EURObservational Research Programme Atrial Fibrillation (EORP-AF) Pilot General Registry. Europace. 2014; 16(3): 308-19. doi: 10.1093/europace/eut373. PMID: 24351881.

3) Hersi A, Abdul-Moneim M, Almous'ad A, Al-Samadi F, AlFagih A, Sweidan R. Saudi Atrial Fibrillation Survey: national, observational, cross-sectional survey evaluating atrial fibrillation management and the cardiovascular risk profile of patients with atrial fibrillation. Angiology. 2015; 66(3): 244-8. doi: 10.1177/0003319714529180. PMID: 24687415.

4) Kannel WB, Benjamin EJ. Status of the epidemiology of atrial fibrillation. Med Clin North Am. 2008; 92: 17-40. doi: 10.1016/j.mcna.2007.09.002. PMID: 18060995, PMCID: PMC2245891.

5) Bin Salih SA, Showlag MS, Al-Qahtani MA, Taha A, Yousuf M, Abdullah M. Clinical characteristics of patients with atrial fibrillation at a tertiary care hospital in the central region of Saudi Arabia. J Family Community Med. 2011; 18(2): 80-4. doi: 10.4103/2230-8229.83374. PMID: 21897916, PMCID: PMC3159233.

6) Emdin CA, Wong CX, Hsiao AJ, Altman DG, Peters SA, Woodward M, et al. Atrial fibrillation as risk factor for cardiovascular disease and death in women compared with men: systematic review and metaanalysis of cohort studies. BMJ. 2016; 532: h7013. doi:10.1136/bmj.h7013. PMID: 26786546 PMCID: PMC5482349.

7) Pancholy SB, Sharma PS, Pancholy DS, Patel TM, Callans DJ, Marchlinski FE. Meta-analysis of gender differences in residual stroke risk and major bleeding in patients with nonvalvular atrial fibrillation treated with oral anticoagulants. Am J Cardiol. 2014; 113: 485-90. doi:10.1016/j.amjcard.2013.10.035. PMID: 24315113.

8) Schnabel RB, Rienstra M, Sullivan LM, Sun JX, Moser CB, Levy D, et al. Risk assessment for incident heart failure in individuals with atrial fibrillation. Eur J Heart Fail. 2013; 15: 843-9. doi:10.1093/eurjhf/hft041. PMID: 23594831, PMCID: PMC3858114.

9) Gutierrez C, Blanchard DG. Diagnosis and Treatment of Atrial Fibrillation. Am Fam Physician. 2016; 94(6): 442-52. PMID: 27637120.

10) Sethi NJ, Feinberg J, Nielsen EE, Safi S, Gluud C, Jakobsen JC. The effects of rhythm control strategies versus rate control strategies for atrial fibrillation and atrial flutter: A systematic review with meta-analysis and Trial Sequential Analysis. PLoS One. 2017; 12(10): e0186856. doi: 10.1371/journal.pone.0186856. PMID: 29073191, PMCID: PMC5658096.

11) Vázquez Ruiz de Castroviejo E, MárquezGarcía A, Fajardo Pineda A, Lozano Cabezas C, Guzmán Herrera M, Ramírez Moreno A, et al. Patterns of clinical presentation of atrial fibrillation in hospitalized patients. Rev Esp Cardiol. 2003; 56(12): 1187-94. doi: 10.1016/S0300-8932(03)77037-7.

12) Chiang CE, Naditch-Brûlé L, Murin J, Goethals M, Inoue H, O'Neill J, et al. Distribution and risk profile of paroxysmal, persistent, and permanent atrial fibrillation in routine clinical practice: insight from the real- 
life global survey evaluating patients with atrial fibrillationinternational registry. Circ Arrhythm Electrophysiol. 2012; 5(4): 632-9. doi: 10.1161/CIRCEP.112.970749. PMID: 22787011.

13) Schnabel RB, Pecen L, Ojeda FM, Lucerna M, Rzayeva N, Blankenberg S, et al. Gender differences in clinical presentation and 1-year outcomes in atrial fibrillation. Heart. 2017; 103(13): 1024-30. doi: 10.1136/heartjnl-2016-310406.

14) Potpara TS, Marinkovic JM, Polovina MM, Stankovic GR, Seferovic PM, Ostojic MC, et al. Genderrelated differences in presentation, treatment and long-term outcome in patients with first-diagnosed atrial fibrillation and structurally normal heart: the Belgrade atrial fibrillation study. Int J Cardiol. 2012; 161(1): 39-44. doi: 10.1016/j.ijcard.2011.04.022.

15) Lip GY, Laroche C, Boriani G, Cimaglia P, Dan GA, Santini M, et al. Sex-related differences in presentation, treatment, and outcome of patients with atrial fibrillation in Europe: a report from the Euro Observational Research Programme Pilot survey on Atrial Fibrillation. Europace. 2015; 17(1): 24-31. doi: 10.1093/europace/euu155. PMID: 24957921.

16) Shehab A, Zubaid M, Bhagavathula AS, Rashed WA, Alsheikh-Ali AA, AlMahmeed W, et al. Sex differences in management and outcomes of patients with atrial fibrillation in the Middle East: Gulf survey of atrial fibrillation events (Gulf SAFE).PLoS One. 2017; 12(5): e0175405. doi: 10.1371/journal.pone.0175405. PMID: 28520719, PMCID: PMC5435140. 